Case Report

\title{
Candida auris and Heart Transplantation. Preoperative Attitude.
}

Raquel López-Vilella ${ }^{1,+, *}$, Alba Cecilia Ruiz-Gaitán ${ }^{2,+}$, José Ricardo Gimeno Costa ${ }^{3,+}$, Elena Marqués-Sulé ${ }^{4,{ }^{+}}$, Rosario Vicente Guillén ${ }^{5,{ }^{\dagger}}$, Javier Pemán García ${ }^{2,{ }^{+}}$, Ignacio Sánchez-Lázaro ${ }^{1,{ }^{\dagger}}$, Luis Almenar Bonet ${ }^{1,+}$

1. Heart Failure and Transplant Unit, Cardiology Department, Hospital Universitario y Politécnico La Fe, Valencia, Spain; E-Mails: lopez raqvil@gva.es; ignaciosanchezlazaro@gmail.com; lualmenar@gmail.com

2. Severe Infection Research Group, Medical Research Institute La Fe, Department of Clinical Microbiology, Hospital La Fe, Valencia, Spain. E-Mails: alba ruiz@iislafe.es; peman jav@gva.es

3. Intensive Medicine, Hospital Universitario y Politécnico La Fe, Valencia, Spain; E-Mail: ricardogimeno55@hotmail.com

4. Physiotherapy Department, University of Valencia, Valencia, Spain; E-Mail: elena.marques@uv.es

5. Anaesthesiology Department, Hospital Universitario y Politécnico La Fe, Valencia, Spain; E-Mail: rosariovicenteguillen@hotmail.com

+ These authors contributed equally to this work.

* Correspondence: Raquel López-Vilella; E-Mail: lopez raqvil@gva.es

Academic Editors: Yasuhiko Sugawara and Kumud K. Dhital

Special Issue: Perspectives on Heart Transplantation

OBM Transplantation

2019 , volume 3 , issue 1

doi:10.21926/obm.transplant.1901053
Received: October 15, 2018

Accepted: February 6, 2019

Published: February 27, 2019

\begin{abstract}
In recent years, several agencies have issued warnings about the spread of Candida auris infections, a multidrug-resistant yeast that is associated with serious infections and with a mortality rate that can reach $70 \%$. This problem is especially serious in transplant recipients receiving intense immunosupressive therapy. There are no established criteria on the
\end{abstract}

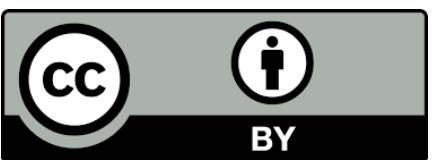

(C) 2019 by the author. This is an open access article distributed under the conditions of the Creative Commons by Attribution License, which permits unrestricted use, distribution, and reproduction in any medium or format, provided the original work is correctly cited. 
relevance of this colonization or on when the transplant should be contraindicated in these cases. Five cases of colonization and two of candidemia have been detected in patients on the heart transplant waiting list. The clinical characteristics and the evolution after the heart transplant of these cases are described in this study, and an action protocol is elaborated in different profiles of patients with colonization and/or infection by $C$. auris on the HT waiting list to help improve risk stratification to Transplant Teams.

\section{Keywords}

Heart transplantation; urgent transplantation; colonization; infection; Candida auris

\section{Background}

Colonization / infection of patients in critical units constitutes a problem that is especially serious in patients waiting for a heart transplant (HT) since they will receive an intense level of immunosuppressive therapy that will last their whole lifetime. Determining the degree of colonization from which the transplant should be contraindicated is a controversial aspect and is far from being resolved. Colonization by Candida has been described in up to 80\% [1] of patients with at least one week of stay in a critical unit. In the last two years, several national and international agencies have issued alerts for the emergence and expansion of infections caused by Candida auris, a multidrug-resistant yeast that is associated with serious infections often in the form of hospital outbreaks in critical care units [2-5]. C. auris can colonize the skin and mucous membranes, and can infect wounds (especially surgical) or cause candidemia and infections with invasion of one or several organs. In case of candidemia or invasion of organs the mortality can reach $70 \%$ [3]. This yeast is also multidrug resistant to the antifungals that are considered first line: it is resistant to fluconazole, and has a variable susceptibility to other azoles, amphotericin B and echinocandins. Recently, the first cases of nosocomial fungemia by $C$. auris have been reported in our center located in the continental Europe [6]. After this, we detected in our center 5 cases of colonization and 2 cases of candidemia due to $C$. auris in patients on the HT waiting list who were hospitalized in the Intensive Care Unit (ICU). Given the impact of $C$. auris colonization or infection in this groupof patients in terms of the possibility of performing HT and short-term prognosis, we describe the clinical and analytical characteristics of these patients, the evolution after HT and the strategy that is used in these cases to help the Transplant Teams to stratify the risk.

\section{Description of Cases}

Table 1 summarizes the characteristics of patients colonized / infected by C. auris before HT. All of these patients were on the urgent HT waiting list with the exception of patient No. 3, who had an elective HT in which post-transplant $C$. auris was isolated. All of them were hospitalized in the ICU. One patient had previously been treated with anti-fungals due to colonization / previous infection by fungi (not $C$. auris) while others had previously been treated with antibiotics for some bacterial infection. All of them had a central venous catheter and bladder catheter, and 6 were carriers of some ventricular assist device (86\%). In the two cases of confirmed candidemia, the blood culture that was finally positive had been extracted before transplantation, but the result 
was obtained within hours-days after the intervention was finished. These two patients received treatment when candidemia was confirmed. Both cases resulted in the death of the patients; the first case by rapidly progressive sepsis (24-48 hours); the second due to respiratory infection and respiratory arrest in the later post-operative period, with no direct relationship with candidemia (Table 1).

Table 1 Clinical-epidemiological characteristics of patients with C. auris colonization or infection.

\begin{tabular}{|c|c|c|c|c|c|c|c|}
\hline & Case 1 & Case 2 & Case 3 & Case 4 & Case 5 & Case 6 & Case 7 \\
\hline Age / Gender & 64 / M & $63 / M$ & $66 / M$ & $59 / M$ & $66 / M$ & $60 / M$ & $55 / M$ \\
\hline Primary etiology & Ischemic DCM & $\begin{array}{l}\text { Post-surgical } \\
\text { valvular } \\
\text { cardiomyopathy }\end{array}$ & Ischemic DCM & Ischemic DCM & Ischemic DCM & $\begin{array}{l}\text { Non-ischemic } \\
\text { DCM }\end{array}$ & $\begin{array}{l}\text { Acute ischemic } \\
\text { heart disease }\end{array}$ \\
\hline $\begin{array}{l}\text { Days at intensive } \\
\text { care unit* }\end{array}$ & 11 & 15 & 30 & 14 & 80 & 24 & 58 \\
\hline Sample / CFU & $\begin{array}{l}\text { Rectal } \\
\text { exudate / } 200 \\
\text { CFU }\end{array}$ & $\begin{array}{l}\text { Tracheal aspirate } \\
\text { / >1000 CFU } \\
\text { Rectal exudate } \\
\text { / >1000 CFU }\end{array}$ & $\begin{array}{l}\text { ECMO wound } \\
\text { exudate and } \\
\text { nasal exudate }\end{array}$ & Rectal exudate & $\begin{array}{l}\text { Oropharyngeal } \\
\text { exudate, rectal } \\
\text { exudate and } \\
\text { tracheal aspirate }\end{array}$ & Rectal exudate & $\begin{array}{l}\text { Urine } 100.000 \\
\text { CFU /ml }\end{array}$ \\
\hline Blood culture & Negative & Negative & Negative & Negative & Negative & Positive & Positive \\
\hline MV /days & Yes / 2 & No & No & No & $\begin{array}{l}\text { Yes/ } \\
\text { Tracheostomy } 30 \\
\text { days }\end{array}$ & No & $\begin{array}{l}\text { Yes/ } \\
\text { Tracheostomy } \\
30 \text { days }\end{array}$ \\
\hline $\begin{array}{l}\text { Central } \\
\text { catheter/days }\end{array}$ & Jugular/11 & Jugular/3 & No & $\begin{array}{l}\text { Jugular/8 } \\
\text { Radial/13 }\end{array}$ & Jugular/6 & $\begin{array}{l}\text { Jugular/3 } \\
\text { Cephalic/16 } \\
\text { Radial/24 }\end{array}$ & Jugular/10 \\
\hline
\end{tabular}




\begin{tabular}{|c|c|c|c|c|c|c|c|}
\hline $\begin{array}{l}\text { Ventricular } \\
\text { assistance / days }\end{array}$ & Levitronix /3 & $\mathrm{ECMO} / 8$ & No & $\mathrm{ECMO} / 4$ & $\begin{array}{l}\text { Long-term LVAD } \\
\text { as Heart Ware }{ }^{\circledR}\end{array}$ & $\mathrm{ECMO} / 23$ & $\begin{array}{l}\text { Levitronix } \\
\text { Centrimag }^{\circledR} / 30\end{array}$ \\
\hline $\begin{array}{l}\text { Previous } \\
\text { tratment with } \\
\text { antobiotics / days }\end{array}$ & $\begin{array}{l}\text { Amikacin/5 } \\
\text { Meropenem/5 }\end{array}$ & $\begin{array}{l}\text { Daptomycin/8 } \\
\text { Ceftriaxone/8 }\end{array}$ & Levofloxacin/5 & Ceftriaxone & Meropenem/10 & $\begin{array}{l}\text { Linezolid/10 } \\
\text { Meropenem/12 } \\
\text { Amikacin/4 }\end{array}$ & $\begin{array}{l}\text { Tigecycline/3 } \\
\text { Meropenem/10 } \\
\text { Amikacin/10 }\end{array}$ \\
\hline $\begin{array}{l}\text { Previous } \\
\text { treatment with } \\
\text { antifungic/days }\end{array}$ & No & Micafungin/8 & No & No & No & No & No \\
\hline $\begin{array}{l}\text { Evolution at } 30 \\
\text { days }\end{array}$ & $\begin{array}{l}\text { Sepsis by } \\
\text { klebsiella } \\
\text { pneumoniae } \\
\text { and death } \\
\text { because of } \\
\text { respiratory } \\
\text { failure at } 90 \\
\text { days }\end{array}$ & $\begin{array}{l}\text { Initially favorable } \\
\text { response. At } 60 \\
\text { days, readmission } \\
\text { by pulmonar } \\
\text { aspergilosis and } \\
\text { death }\end{array}$ & $\begin{array}{l}\text { Torpid wound } \\
\text { evolution } \\
\text { (ECMO). Alive }\end{array}$ & Favorable & $\begin{array}{l}\text { Death at } 60 \text { days } \\
\text { because of severe } \\
\text { myopathy and } \\
\text { aspiration with } \\
\text { respiratory failure }\end{array}$ & $\begin{array}{l}\text { Refractory septic } \\
\text { shock and MOF. } \\
\text { Death } 48 \text { hours } \\
\text { after HT }\end{array}$ & $\begin{array}{l}\text { Death at } 30 \\
\text { days because of } \\
\text { respiratory } \\
\text { sepsis and } \\
\text { respiratory } \\
\text { failure }\end{array}$ \\
\hline
\end{tabular}

* Before first isolation

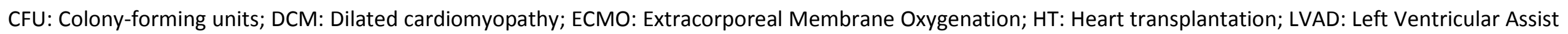
Device; M: Male; MOF: Multi-organ failure; MV: Mechanical ventilation. 
We have established an action protocol (Figure 1) in different profiles of patients on the HT waiting list with colonization and / or infection due to $C$. auris. This paper aims to present our experience and protocol so that it can be adapted to other similar centers (Figure 1):

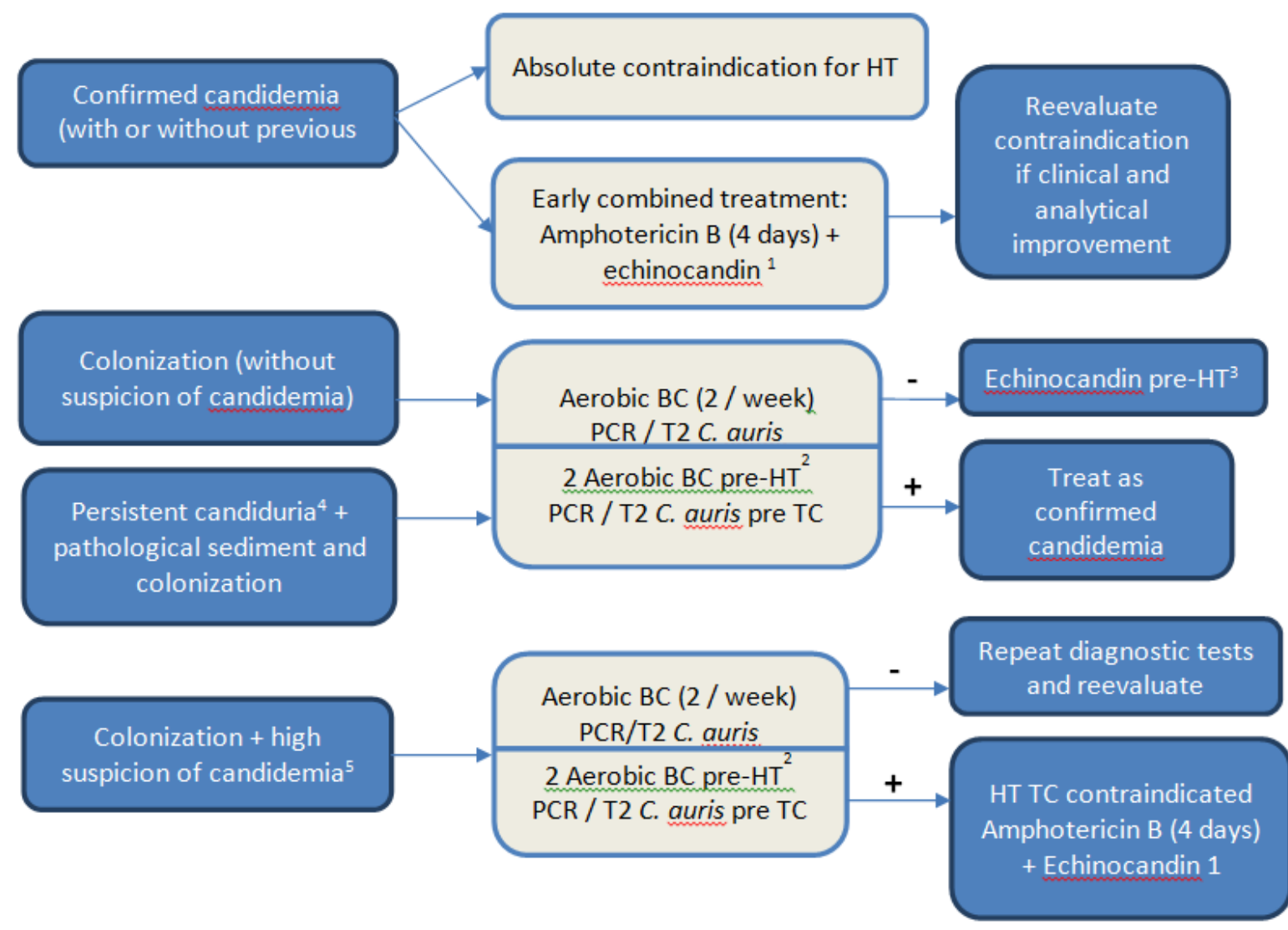

Figure 1 Protocol in different profiles of patients on the heart transplantation waiting list with colonization and / or infection due to $C$. auris.

$\left({ }^{1}\right.$ Continue with echinocandin and reassess contraindication if there is a clinical and analytic improvement, with at least 2 negative blood cultures separated by 72 hours.

${ }^{2}$ From peripheral blood and central line.

${ }^{3}$ Treatment with echinocandin begins at the intensive care unit only if transplant is immediate, once organ validity is confirmed. It is maintained for 7 days.

${ }^{4}$ Candiduria remains after removing/changing urinary catheter.

${ }^{5}$ Clinical suspect of sepsis (fever, hypotension, elevation of acute phase reactants, etc.)+ biomarkers suggesting infection, accompanied by rectal colonization and colonization in two more foci.

BAS: Bronchial aspirate; BC: Blood culture; HT: Heart transplantation; PCR/T2 Candida: Detection panel of Candida; TA: Tracheal aspirate.)

- Surveillance cultures are routinely performed at the patient's entrance and once a week in the ICU. Samples of oropharyngeal exudate and rectal exudate are performed. In patients with mechanical ventilation, a bronchoaspiration sample is also sent. These cultures are aimed at systematically detecting the presence of multidrug resistant microorganisms, such as methicillinresistant Staphylococcus aureus and multidrug resistant gram-negative bacilli: Enterobacteriaceae and Pseudomonas that produce extended-spectrum beta-lactamases (ESBL) and carbapenemases. 
- $\quad H T$ is contraindicated if candidemia by $C$. auris is confirmed. In these cases, early treatment with liposomal amphotericin B ( $5 \mathrm{mg}$ per $\mathrm{Kg}$ of weight per day) and an echinocandin are initiated. The most commonly used echinocandins are: caspofungin (loading dose of $70 \mathrm{mg}$ followed by 50 $\mathrm{mg}$ / day), anidulafungin (loading dose of $200 \mathrm{mg}$ followed by $100 \mathrm{mg} /$ day), micafungin (100 mg / day). Isavuconazole is also used (loading dose of $200 \mathrm{mg} / 8 \mathrm{~h}$ during the first 48 hours followed by $200 \mathrm{mg} /$ day). We maintain a dual therapy with liposomal amphotericin B and echinocandin for 4 days, and later maintaining only the echinocandin. This is an empirical approach, agreed by a multidisciplinary team of professionals in infectious diseases, microbiology, intensive care and cardiologists. In the case of clinical and analytical improvement with two negative blood cultures separated by 72 hours, the case can be re-evaluated. There are some cases in which $C$. auris has reappeared in blood cultures after more than one negative blood culture and, on the other hand, we cannot forget that these patients are going to receive intense immunosuppression, especially in the early stages of heart transplantation. Therefore, it is controversial that the negativization of cultures necessarily implies the eradication of the infection. Therefore, in these cases it is preferable, if possible, to postpone transplant as far as possible.

- If patients who are candidates for HT are found with colonization but with no candidemia, then no initial prophylaxis is established. However, a PCR (Polymerase chain reaction)/ T2 will be performed at the time when a compatible donor emerges. This is a technique that allows to quickly identify the causative agent of candidemia directly from a blood sample (without a previous culture) with a predictive value that reaches $99 \%$. If PCR is negative, empirical therapy with a first dose of echinocandin is administered just before HT with the same doses detailed in the previous paragraph. This treatment is maintained for at least 7 days, suspending it after 2 negative control blood cultures separated from each other for 72 hours. If PCR is positive, treatment is started as if it were a confirmed candidemia (see previous paragraph).

- Colonization by $C$. auris in the urine requires an immediate replacement of the bladder catheter. In patients who are candidates for a HT with a persistent candiduria due to $C$. auris despite the replacement of the bladder catheter, AmB deoxycholate bladder irrigation at $50 \mathrm{mg} / \mathrm{L}$ sterile water daily for 5 days may be useful for the treatment of cistitis due to fluconazoleresistant species (weak recommendation; low-quality evidence) [7]. In this group of patients, as in the previous profile, PCR is also performed for $C$. auris at the moment when there is a compatible donor, and the attitude described in the previous point is carried out. If the number of colony forming units exceeds one hundred thousand, it is considered urinary infection and, in addition to establishing anti-fungal treatment as previously described, it is preferable, if possible, to postpone heart transplantation.

- C. auris in whom there is a high suspicion of candidemia, determined by clinical suspicion of sepsis (fever, hypotension...) and the presence of biomarkers suggestive of infection: PCR, leukocytes, percentage of neutrophils and procalcitonin... This situation in our experience occurs more frequently when the colonization is rectal and in other foci (urine, oropharyngeal exudate, bronchial aspirate or tracheal aspirate). The management in these cases is similar to that of the other colonized patients, but the high suspicion of candidemia, although not confirmed, requires strict microbiological control, periodically repeating the diagnostic tests that are considered necessary and reassessing the patient's situation on a daily basis. If there is a high suspicion of candidemia, despite not being confirmed, we must take into account the difficulty in detecting and identifying this microorganism, and postpone transplantation. 
Daily skin decolonization of colonized patients with aqueous chlorhexidine $2 \%$ wipes is performed. However, some patients presented skin reactions to the aqueous solution. In all cases, it is important to avoid intrahospital transmission by means of barrier measures, and the early isolation of all patients infected or colonized by $C$. auris until having at least 3 consecutive negative cultures once per week [8].

\section{Discussion}

The distribution of Candida species has changed in recent decades, with a substantial appearance of non-albicans Candida species, especially in relation to the use of prophylactic antifungal treatment in high-risk populations [9]. In the last two years, several national and international agencies have issued alerts for the emergence and expansion of infections caused by Candida auris. The actual incidence of $C$. auris infections is probably underestimated, since the usual techniques do not correctly identify this species. Even so, from the first case described in 2009 [10], cases of $C$. auris colonization and infection have been increasing in tertiary hospitals in different countries.

Given the high percentage of transplants performed in urgent code [11], and the increasing use of circulatory / ventricular assist devices, transplant patients frequently present prolonged pretransplant stays in critical units. In the cases that we present, all of the patients were hospitalized in a critical unit for refractory cardiogenic shock, with an average stay in this unit of 33 days. This reflects that, despite the relatively short waiting times for urgent heart transplantation in Spain, as in all transplant programs, the number of donors is low, and the patients, usually in complex clinical situations, can spend long periods of time in the ICU before receiving an urgent HT. All of the patients in our series had some circulatory / ventricular assist device, with the exception of patient No. 3, who had an elective HT in which post-transplant $C$. auris was isolated. Patient No. 3 had previously been assisted by Extracorporeal Membrane Oxygenation (ECMO), who had already withdrawn. All of the patients had been previously treated with antibiotics, which implies the high frequency of colonization +/- infection from a certain number of days in this type of units.

Patient No.2 had also received treatment with prophylactic anti-fungal (micafungin), because prior to the isolation of $C$. auris, had presented with colonization by other candida species in the oropharynx along with elevation of acute phase reactants in blood tests.

In the two cases of confirmed candidemia, the blood culture that was finally positive had been extracted before transplantation, but the result was obtained within hours-days after the intervention was finished. This fact reflects the difficulty in diagnosing this type of candida. The diagnosis of candidiasis by $C$. auris requires: clinical suspicion and image and laboratory data compatible with invasive candidiasis, along with the cultures of the clinical samples and the detection of fungal biomarkers. This species of candida is not identified by the usual commercial techniques based on biochemical characteristics. Therefore, it is necessary to confirm the identification by DNA sequencing of all the isolates resistant to fluconazole [3,12]. At present, mass spectrometry and DNA sequencing are the most reliable methods for identification [5, 13]. In our center, all Candida isolates were identified by phenotypic and biochemical characteristics, proteomic profiling and DNA sequencing technology. Proteomic profiles were obtained and evaluated by VITEK MS RUO version (bioMérieux) according to the manufacturer's instructions. Definitive identification was performed by sequencing of internal transcribed spacer (ITS) using 
the primers ITS3 -ITS4 and ITS2 -ITS5 as previously described (ref bellamein); sequencing was performed using GenomeLabTM GeXP (Beckman Coulter, Fullerton, CA, U . S . A .) equipment and the sequences obtained were compared with those in Microbial Genomes BLAST (http://www.ncbi.nIm.nih.gov/guide/sequence-analysis/) [14]. The recent introduction of the Candida T2 panel allows rapid identification of the causative agent of candidemia directly from a blood sample, without a previous culture, with a predictive value that reaches $99 \%$.

Regarding the fungal biomarkers used for diagnosis (for example, Candida antigen detection, beta-glucan detection, antibodies ...), although some biomarkers such as antimicelium are available, they are not routinely performed. The available PCRs used corresponds to the commercial kits with CEM such as the Auris ID ${ }^{\circledR}$, which allows for the identification of $C$. auris in whole blood, blood culture and swabs with a good performance. In any case, it is important to rule out infection in patients with colonization, since a candidemia rate of up to $18 \%$ is described among colonized patients [15]. These values are high and represent a serious risk for critical patients, and make it necessary to reliably rule out the presence of candidemia if the HT option is considered.

In relation to the treatment, all of our blood isolates independently of susceptibility method was used, were resistant to fluconazole (minimum inhibitory concentration (MIC) $>256 \mu \mathrm{g} / \mathrm{mL}$ ) and voriconazole (MIC $>32 \mu \mathrm{g} / \mathrm{mL}$ ). Among echinocandins, all isolates were susceptible to anidulafungin and micafungin (MIC $\leq 0.5 \mathrm{mg} / \mathrm{L}$ and $\leq 0.12 \mathrm{mg} / \mathrm{L}$, respectively). All $C$. auris candidemia were initially treated with an echinocandin following susceptibility report and the recommendations of Infectious Diseases Society of America guidelines [7]. In the cases where patient did not respond clinically (for example lack of clearance) a combination of antifungals with a lipid formulation of amphotericin B was considered. None of our C. auris isolates had developed echinocandin resistance. Two patients with confirmed candidemia was given liposomal amphotericin B ( $5 \mathrm{mg}$ per kg of weight per day) and aniulafungin (loading dose of $200 \mathrm{mg}$ followed by $100 \mathrm{mg}$ per day). Both cases resulted in the death of the patients; the first case by rapidly progressive sepsis (24-48 hours); in the second due to respiratory infection and respiratory arrest in the later postoperative period, with no direct relationship with candidemia.

This registry is retrospective, however, it is the first study that comments on the pre-transplant attitude of patients colonized by $C$. auris and details a concrete protocol of action.

In conclusion, colonization / infection by $C$. auris in critical units is not uncommon, and in candidates for heart transplantation requires early detection and treatment. On the other hand, it is necessary to reconsider whether colonization is a contraindication for HT because of the high probability of post-transplant infection.

\section{Acknowledgments}

Acknowledge the people or organization(s) that have technically supported this work, excluding fund provider.

\section{Author Contributions}

Raquel López-Vilella: Writing, revision, correction.

Alba Cecilia Ruiz Gaitán: Revision, correction.

José Ricardo Gimeno Costa: Revision, correction. 
Elena Marqués-Sulé: Revision, correction.

Rosario Vicente Guillén: Revision, correction.

Javier Pemán García: Revision, correction.

Ignacio Sánchez-Lázaro: Writing, revision, correction.

Luis Almenar Bonet: Writing, revision, correction.

\section{Competing Interests}

The authors have declared that no competing interests exist.

\section{References}

1. Vincent JL, Rello J, Marshall J, Silva E, Anzueto A, Martin CD, et al. International study of the prevalence and outcomes of infection in intensive care units. JAMA. 2009; 302: 2323-2329.

2. Io Foundation [Internet]. Spain: foundation; 2017. Available in: http://fundacionio.org/viajar/enfermedades/candida\%20auris.html

3. Clancy CJ, Nguyen NH. Emergence of Candida auris: An international call to arms. Clin Infect Dis. 2017; 64: 141-143.

4. Lockhart SR, Etienne KA, Vallabhaneni S, Farooqi J, Chowdhary A, Govender NP, et al. Simultaneous emergence of multidrug-resistant Candida auris on 3 con-tinents confirmed by whole-genome sequencing and epidemiological analyses. Clin Infect Dis. 2017; 64: 134-140.

5. Centers for Disease Control and Prevention Clinical Alert to U.S. Healthcare Facilities - June 2016 Global Emergence of Invasive Infections Caused by the Multidrug-Resistant Yeast Candida auris. Available in: https://www.cdc.gov/fungal/diseases/candidiasis/candida-aurisalert.html

6. Ruiz Gaitán AC, Moret A, López Hontangas JL, Molina JM, Aleixandre López Al, Cabezas AH, et al. Nosocomial fungemia by Candida auris: First four reported cases in continental Europe. Rev Iberoam Micol. 2017; 34: 23-27.

7. Pappas PG, Kauffman CA, Andes DR, Clancy CJ, Marr KA, Ostrosky-Zeichner L, et al.' Clinical practice guideline for the management of candidiasis: 2016 update by the infectious diseases society of America. Clin Infect Dis. 2016; 62: e1-e50.

8. Public Health England Guidance for the laboratory investigation, managementand infection prevention and control for cases of Candida auris. Available in: https://assets.publishing.service.gov.uk/government/uploads/system/uploads/attachment d ata/file/637685/Updated Candida auris Guidance v2.pdf

9. Chowdhary A, Voss A, Meis JF. Multidrug-resistant Candida auris: 'New kid on the block' in hospital-associated infections?. J Hosp Infect. 2016; 94: 209-212.

10. Satoh K, Makimura K, Hasumi Y, et al. Candida auris sp. nov., a novel ascomycetous yeast isolated for the external ear canal of an inpatient in a Japanese hospital. Microbial Inmunol. 2009; 53: 41-44.

11. González-Vílchez F, Segovia Cubero J, Almenar L, et al. Registro Español de Trasplante Cardiaco. XXVII Informe Oficial de la Seccioón de Insuficiencia Cardiaca y Trasplante Cardiaco de la Sociedad Española de Cardiología (1984-2015). Rev Esp Cardiol. 2016; 69: 1071-1082 
12. Calvo B, Melo AS, Perozo-Mena A, Hernandez M, Francisco EC, Hagen F, et al. First report of Candida auris in America: Clinical and microbiological aspects of 18 episodes of candidemia. J Infect. 2016; 73: 369-374.

13. Kathuria S, Singh PK, Sharma C, Prakash A, Masih A, Kumar A, et al. Multidrugresistant Candida auris misidentified as Candida haemulonii: Characterization by mtrix-assisted laser desorption ionization-time of flight mass spectrometry and DNA sequencing and its antifungal susceptibility profile variability by Vitek 2, CLSI broth microdilution, and Etest method. J Clin Microbiol. 2015; 53: 1823-1830.

14. Bellemain E, Carlsen T, Brochmann C, Coissac E, Taberlet P, Kauserud H. ITS as an environmental DNA barcode for fungi: An in silico approach reveals potential PCR biases. BMC Microbiol. 2010; 10: 189.

15. Schelenz S, Hagen F, Rhodes JL, Abdolrasouli A, Chowdhary A, Hall A, et al. Firsthospital outbreak of the globally emerging Candida auris in an European hospital. Antimicrob Resist Infect Control. 2016; 5: 35.

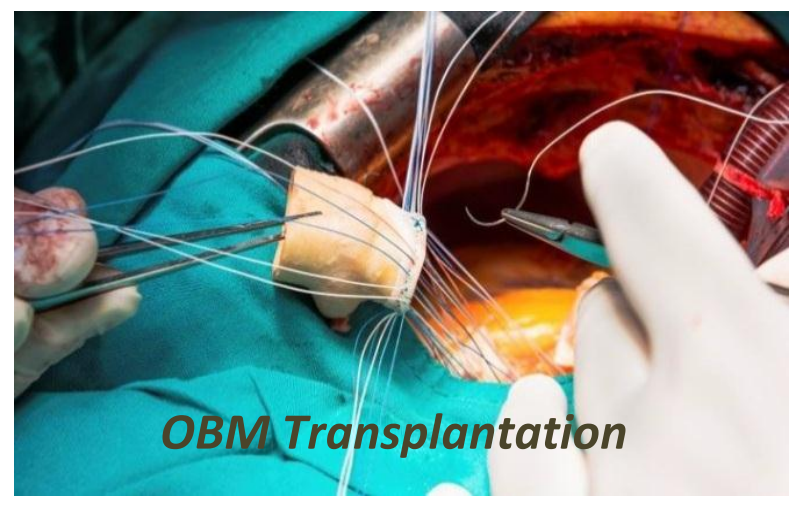

Enjoy OBM Transplantation by:

1. Submitting a manuscript

2. Joining in volunteer reviewer bank

3. Joining Editorial Board

4. Guest editing a special issue

For more details, please visit:

http://www.lidsen.com/journals/transplantation 